\title{
Information overload challenges pandemic control
}

- Cite as: CMAJ 2021 August 9;193:E1222-3. doi: 10.1503/cmaj.1095955

Posted on cmajnews.com on July 26, 2021

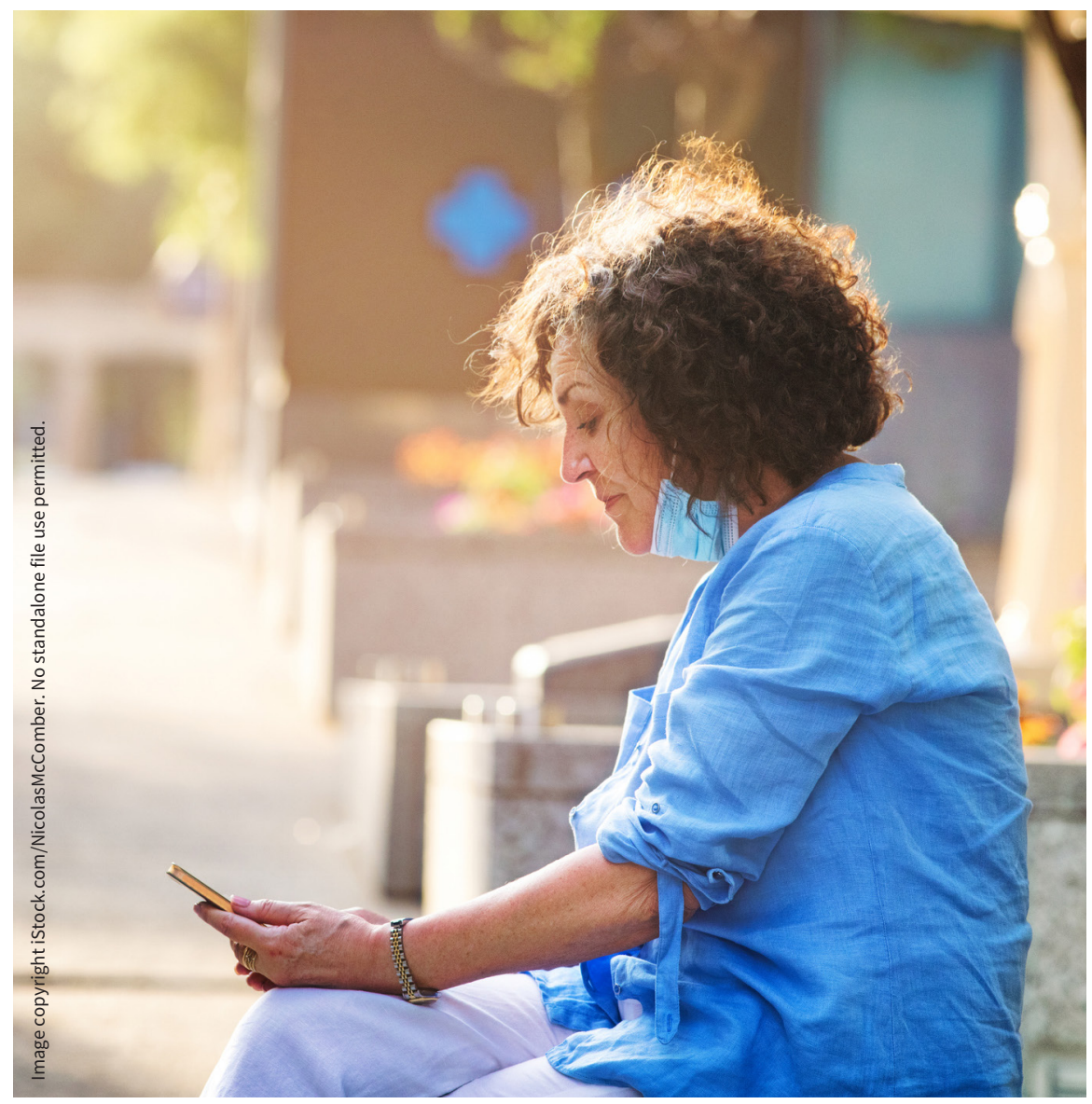

Tuning out COVID-19? Even health workers may struggle with pandemic "message fatigue."

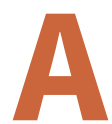

s the pandemic wears on, some experts caution that growing desensitization to public health messages may complicate ongoing efforts to curb SARS-CoV-2.

A recent study in the Journal of Medical Internet Research Infodemiology analyzed anxiety levels expressed in COVID-19 news articles and related social media posts over 11 months. Researchers from the University of California, Davis, found that high levels of anxiety expressed early in the pandemic waned over time, even as the death toll increased.

According to the authors, their findings suggest that people are becoming desensitized to information about COVID-19 - which may leave them out of the loop when threat levels change.

"Our study shows a need to delve deeper into how to re-sensitize the public and motivate them to take action in the face of an ongoing emergency," writes lead author Hannah Stevens. "If another health crisis occurred today, or COVID19 takes another turn for the worse, it is essential for public health officials to consider that they are communicating to a desensitized public."

Overexposure to repetitive messages or "message fatigue" has been linked with disengagement and other problems for public health campaigns aimed at reducing obesity and promoting safer sex.

During the pandemic, at least one small American study found that similar negative reactions to repetitive COVID-19 messages were associated with lower levels of adherence to precautions like handwashing and physical distancing.

Meanwhile, public health experts and media reports have often cited message fatigue as a factor in outbreaks alongside general weariness with pandemic measures.

"Desensitization to messages is complex and builds with time," said Larry Svenson, an associate professor in the faculty of medicine at the University of Alberta and provincial health analytics officer.

Fear can be motivating in the short term, but some level of numbing is necessary for coping with prolonged threats. Like the constant beeping of pumps and monitors in intensive care, even emergency alerts can eventually become background noise.

As the effects of COVID-19 snowball, "it can be difficult to realize that all those statistics on deaths, hospitalizations, ICU admissions and postponed surgeries represent real people," Svenson said. "Not seeing the faces behind those 
numbers... erodes our compassion in ways we may not realize."

Health workers may be particularly susceptible to message fatigue given their constant exposure to infection control advice and reminders.

During the H1N1 pandemic in the late 2000s, American primary care providers reported feeling overwhelmed by the volume of emails from public health authorities and health care organizations. Only one-third visited government or institutional websites at least weekly to keep up with changing advice. And although most physicians were knowledgeable about initial pandemic guidance, fewer than half stayed up to date as that guidance changed.

A randomized study of 528 health care providers published in the wake of the H1N1 pandemic found that their odds of recalling public health messages dropped by $41 \%$ with each additional message received in a week. According to the authors, "information overload" may hamper health workers' response to public health emergencies when message volumes may be higher.

Despite heavy emphasis on hand hygiene and proper use of protective gear to curb the spread of SARS-CoV-2, health workers' compliance with these measures has been inconsistent, even early in the pandemic when anxiety about COVID-19 was high.

One Italian study found hospital workers' handwashing habits improved only in intensive care units, where the threat posed by the virus was most obvious. Even in intensive care, handwashing rates ranged from $42 \%$ among resident physicians to $77 \%$ among nurses. Other studies from Israel and the United
States observed low rates of health workers correctly donning and doffing protective gear, including after receiving training.

Overwhelming workloads, understaffing and burnout likely factor into health workers' ability to absorb and follow pandemic advice. "Health care workers are tired and will continue to be tired as the pandemic continues," said Svenson.

Frequent requests to participate in COVID-19 research may also contribute to information overwhelm. One mixedmethods study found British health workers received numerous invitations to complete surveys that were often redundant and methodologically questionable.

However, some experts reject the notion that message fatigue and general weariness with pandemic measures lead to poor compliance. According to British psychologists Stephen Reicher and John Drury, such framing "feeds into a widespread narrative of blame whereby the spread of infections is explained in terms of those who choose to break the rules, rather than failures of public health response."

"One also avoids acknowledging the failures of government to provide the support necessary to follow the rules," they argued in The BMJ.

Canadians surveyed by Impact Canada late last year reported high levels of compliance with public health precautions despite feeling tired of those measures.

According to British Columbia's Ministry of Health, by now most people know the drill when it comes to reducing their risk of infection. "We know people are tired and are ready to get back to the things they love."

But widespread vaccination and the emergence of more transmissible variants of the virus add new variables that may complicate Canada's pandemic calculus.

Some researchers suggest that fewer, clearer messages focused on narratives, rather than numbers, may help cut through message fatigue as provinces continue to lift restrictions.

Earlier this year, Ontario's COVID-19 Science Advisory Table recommended moving beyond "more of the same" messages about avoiding risk to emphasize well-being. That includes highlighting activities that can be done safely, underscoring that "every effort counts," changing up signs and other media, and using humour and incentives to promote positive action.

"The science of getting people to start something new is different from the science of getting them to continue positive behaviours," the advisory group explained. "Promising strategies to increase effective masking and physical distancing include persuasion, enablement, modelling the behaviour, and clear education."

\section{Diana Duong, CMAJ}

Content licence: This is an Open Access article distributed in accordance with the terms of the Creative Commons Attribution (CC BYNC-ND 4.0) licence, which permits use, distribution and reproduction in any medium, provided that the original publication is properly cited, the use is noncommercial (i.e., research or educational use), and no modifications or adaptations are made. See: https://creativecommons.org/licenses/by-nc -nd/4.0/ 